\title{
Australian biodiversity via its plants and marine organisms. A high-throughput screening approach to drug discovery*
}

\author{
Ronald J. Quinn ${ }^{1, \neq}$, Priscila de Almeida Leone ${ }^{1}$, Gordon Guymer², \\ and John N. A. Hooper ${ }^{3}$ \\ ${ }^{1}$ AstraZeneca R\&D Griffith University, Brisbane, Queensland, 4111, Australia; \\ ${ }^{2}$ Queensland Herbarium, Brisbane, Queensland, 4066, Australia; ${ }^{3}$ Queensland \\ Museum, Brisbane, Queensland, 4101, Australia
}

\begin{abstract}
High-throughput screening (HTS) of extracts of Australian plants and marine organisms commenced in our laboratory in 1994. The biota collections commenced in late 1993. The collection has in excess of 30000 biota samples including over 16000 biota samples of vascular plants, algae, and macro fungi from Queensland, and over 4000 marine invertebrates from Australian waters. The plant collection represents $\sim 9 \%$ of the world species diversity of higher plants, with representation from $73 \%$ of the world's plant families. The marine collection contains $\sim 10 \%$ of the world diversity of sponges, $\sim 10 \%$ of the world diversity of ascidians, and $\sim 5 \%$ of the world diversity of soft corals and gorgonians.

The lecture will highlight some of the advances to knowledge about Australian biodiversity as a result of the HTS project, discuss drug discovery using HTS, and give some examples of the chemistry arising from the screening of the extracts.
\end{abstract}

Australia is one of 12 megadiverse countries (Mexico, Colombia, Ecuador, Peru, Brazil, Zaire, Madagascar, China, India, Malaysia, Indonesia, Australia). These countries have greater than $70 \%$ of world biodiversity [1]. Australia is a party to the Convention on Biological Diversity, which states that participating countries have sovereign rights over their own biological resources.

Historically, natural products have been a significant contributor to drug discovery. In 1996, eight of the 20 top-selling pharmaceuticals were natural product-derived. In the period 1983-1994, 203 out of the 520 NCEs introduced worldwide were derived from natural sources [2]. There has been a high success rate of NPS in oncology with $61 \%$ of NCEs introduced between 1983-1994 being natural product-derived [2]. Taxol/taxotere (from plants) has achieved combined No. 1 sales in oncology. Diversity analysis by Bayer of its 350000 -compound synthetic library plus other databases indicated $40 \%$ of natural products were not represented by the investigated synthetics [3]. Complementarity of diversity is obtained by a combined compound and natural product library.

It is estimated that the diversity of living organisms on Earth is between 5 and 100 million species (only 1.4 million have actually been described). Bacteria and arthropods make up the vast majority of this biodiversity ( $90 \%$ ). It has also been estimated that $90-99 \%$ of bacteria cannot be cultured using techniques available today. Natural product drug discovery programs have traditionally targeted those organisms that can be easily collected or cultured, and include plants, dominant ( $>1 \mathrm{~kg}$ ) marine organisms, and culturable microbes. The AstraZeneca-Griffith University (AZGU) collection asset com-

\footnotetext{
${ }^{*}$ Lecture presented at the $3^{\text {rd }}$ IUPAC International Conference on Biodiversity (ICOB-3), Antalya, Turkey, 3-8 November 2001. Other presentations are presented in this issue, pp. 511-584.

${ }^{\ddagger}$ Corresponding author
} 
prises 35220 biota and includes collections of vascular plants, algae, and macro fungi (24 017) from Queensland (18 732), Southern China (6314), and Papua New Guinea (2501), marine invertebrates from Australian and Antarctic waters (5871), soil and aquatic fungal extracts from India (1802).

The plant collection represents $\sim 9 \%$ of the world species diversity of higher plants, with representation from $73 \%$ of the world's plant families. The marine collection contains $\sim 10 \%$ of the world diversity of sponges, $\sim 10 \%$ of the world diversity of ascidians, and $\sim 5 \%$ of the world diversity of soft corals and gorgonians.

The natural product drug discovery program in our laboratory consists of plant and marine collection, extraction, high throughput screening of the extracts, identification of active extracts, bioassaydirected fractionation, isolation of the active constituents, structure elucidation, and lead optimization using medicinal chemistry. At the commencement of the project we envisaged the need to isolate only $1 \mathrm{mg}$ of material for structure elucidation and biological evaluation in the screen of interest.

The requirement for a small amount of isolated compound established the logistics of our natural product drug discovery project. Small-scale extraction and chromatography were integral to the approach. The gains in efficiency with miniaturization have been substantial, allowing the isolation of over 600 bioactive compounds.

In order to be successful in any screening operation, the vast amount of data generated by HTS of extracts, fractions, or compounds must be harnessed. We have opted to write our own software. This decision was based on evaluation of commercial packages and a decision that in-house development would allow as good or better customization in the initial stages but, critically, would allow easier adaptation to future unforeseen requirements. The software package, HiTbaSe, has served us extremely well. HiTbaSe is a client/server-based system with graphical client software for data entry and query. All information is stored in SQL tables on an Oracle server. Assays, experiments, and plate templates can be set up via an intuitive user interface. With the aid of a user-configurable import system HiTbaSe reads raw screening data from any file format. HiTbaSe provides a comprehensive quality control and decision-making environment via its "Activity Centre". Hit Selection is performed by "Active Search", a powerful visualization and filtering interface. The system also tracks sample status and status history, providing powerful list management options. The Plate Wizard provides a logical way of submitting low- to high-volume follow-up experiments. Performance is high due to optimized ADO connectivity directly with Oracle. A performance test, of rank ordering a 140000 well screen in descending order took $35 \mathrm{~s}$-on a 400-MHz Sun Enterprise 250 Oracle server.

HTS has focused on receptors, enzymes, and mechanism-based cellular assays in the area of cardiovascular, respiratory, inflammation, gastrointestinal, pain control, CNS, infection, and oncology.

HTS commenced in July 1994. Continuous screening, where a panel of around 20 screens was assayed each year against 10000 extracts was implemented from 1994-1997. The process identified potential lead compounds, however this required a screen lifetime of two years to evaluate 20000 extracts. This was not in pace with the need for rapid identification of leads. The most interesting or potent extract may not be identified until the later part of the screening.

In 1997, with the extract collection at 30000 extracts, continuous screening was replaced by campaign screening. Campaign screening allowed the entire extract library to be screened in a semi-automated manner within four weeks. Between 1997 and 2000, the extract library increased greater than threefold while the time to assay the extract library either remained at the 1997 level or in most cases was able to be decreased. This was accomplished by the judicious purchase of state-of-the-art robotic liquid handling and assay building workstations that allow 100 384-well plates per day (depending on screen technology). Four robotic workstations (assay building stations) are in place.

The first 100000 extract campaign was achieved in May 2000. In 2000, the number of wells (assay points) achieved in primary screening was $273 \%$ of the number in 1999. Also in 2000, the number of extracts per screen increased substantially and was $244 \%$ of the 1999 figure.

In terms of biodiversity conservation, it is valuable to examine the effect of this miniaturization. A comparison of biodiversity discovery programs of the 1970s with current efforts also highlights the 
increased biodiversity conservation value of today's research. The Roche Research Institute of Marine Pharmacology (RRIMP) operated in Sydney between 1974 and 1981. Sample collections were $10 \mathrm{~kg}$ of wet sponge. This amount was a function of the screening, isolation, and structure elucidation requirements. Screening was based on animal models and isolated tissue preparations. Six extracts originating from two species were screened each week against a panel of screens. In total, around 2100 extracts were screened over seven years against a panel of screens. The AZGU program collects around $100 \mathrm{~g}$ of biota. Extraction of $200 \mathrm{mg}$ of biota provides sufficient extract for screening against receptors, enzymes, and mechanism-based cellular targets. This has allowed the screening of 100000 extracts against the targets.

In 96-well format, screening the extract from $100-1000 \mu \mathrm{g}$ of sample per well (usually $200 \mu \mathrm{L}$ ) allows $200 \mathrm{mg}$ of sample to give 2000-200 assays points. The more recent introduction of 384-well format allowed a reduction of assay volume to around $50 \mu \mathrm{L}$, so that $200 \mathrm{mg}$ of biota at $25-250 \mu \mathrm{g}$ per well provides $8000-800$ assay points.

\section{New knowledge of the marine fauna}

The sea contains a much greater potential source of genetic diversity than does the land, with a much larger variety of life forms (body plans, or phyla) that live there. Of the 28 marine phyla about 33000 species have been described from the Australian EEZ (excluding vertebrates apart from fishes) (ABRS, 2001). This fauna currently known to science is estimated to represent less than one-third of the total number of species actually living in Australian waters, which in turn is also estimated to comprise about $30 \%$ of the world's marine fauna.

The Australian marine biota is also highly unique, with many species not found anywhere else in the world. It has both Tethyan and Gondwanan origins, with the former responsible for the mainly tropical species that are supposedly widely distributed throughout the Indo-west Pacific (an equivocal claim that still remains largely untested), and the latter for the more highly endemic, mainly southern faunas. Moreover, in the transition zones, where these northern and southern faunas overlap, there has been a substantial mixing of species that has produced many endemic regional marine faunas, on both the east and west coasts of the continent. Similarly, there are over 60 marine bioregions in the Australian EEZ [4], each characterized by particular suites of biota and physical-chemical features that set them apart from other regions, together representing a huge diversity of ecosystems. Consequently, as for the terrestrial fauna and flora, the Australian marine biota is also megadiverse. However, despite these claims, we still know very little about our marine biota, with about $40 \%$ of marine families lacking even basic data.

Over the past decade we have come a lot closer to understanding the magnitude and diversity of some of these marine phyla, thanks largely to biodiscovery/bioprospecting for new chemical compounds. Since the AZGU "biodiscovery" collection programs commenced in 1993 our knowledge of the biodiversity of sessile marine invertebrates (animals fixed to the seabed) has increased phenomenally, especially of the lower phyla containing sponges, soft corals, gorgonians, and bryozoans, as well as members of the higher invertebrates, viz., tunicates, or ascidians. These taxa are among the most interesting and bioactive targets for pharmaceutical research worldwide [5]. During the AZGU program over 8000 marine specimens have been collected, of which 5858 specimens (weighing more than $100 \mathrm{~g}$ wet wt) were used for screening operations. These collections consist of about 3500 species and 2005 species, respectively, collected from over 1000 individual sites throughout tropical Australia. The proportion of specimens (and species) for the target phyla is as follows:

- Phylum Porifera. Collections of sponges currently consist of 4420 specimens $>100 \mathrm{~g}$ wet wt, representing 1247 species. Additional collections of $\sim 2200$ specimens $<40 \mathrm{~g}$ wet wt have also been made during this program, representing $\sim 1500$ species, mainly the small encrusting and cryptic species that were too small to produce adequate chemical extracts for screening operations. 
- Phylum Cnidaria. These collections consist of 681 specimens of soft corals, gorgonians, jellyfish, anemones, and related taxa, consisting of 292 species. Collections of cnidarians for the AZGU program ceased last year on the basis that their chemical diversity was predictable and unproductive in screens. Few hard corals have been collected given their low proportion of living tissue to inorganic skeleton, physical difficulty associated with making extracts, and conservation issues associated with collecting scleractinian corals.

- Phylum Bryozoa. To date only a few of these species, known as moss animals or lace corals, have been collected, consisting only of 31 specimens in seven species. Although bryozoans are a potentially very productive group of sessile marine invertebrates, with a high proportion of bioactive compounds known worldwide, the tropical fauna contains species of generally small body weight, and with a low proportion of living tissue to inorganic skeleton, such that it is extremely difficult to collect specimens of $>100 \mathrm{~g}$ wet wt.

- Phylum Chordata. In the same phylum as humans, there is a group of sessile animals called tunicates, ascidians, or sea squirts, that also have a demonstrated track record as being productive sources of bioactive compounds. During the AZGU program 397 specimens of ascidians, in 156 species, have been collected, with an estimated 500 or more species living in Australian waters still unsampled to date, many of which are important components to the encrusting and cryptic coral reef communities.

Several other phyla, including mollusks, deep-sea fishes, crustaceans, and echinoderms have also been collected during this program, with 324 specimens in 303 species sampled to date. By-and-large, however, these have not generally yielded any bioactivity of interest to the current program, and their collection is more opportunistic, especially unusual species in unique habitats.

Sponges, in particular, remain key targets for the AZGU biodiscovery program, providing a major source of new chemical structures and bioactive leads [5]. They are also the most interesting from a scientific perspective, containing a huge diversity of largely unnamed (new) taxa worldwide, with a particularly rich and unique Australian fauna, and containing representatives of living fossils - taxa left over from the Mesozoic era, apparently still alive and well.

Since 1993, our knowledge of this sponge fauna has increased threefold, and more significantly, most of the new discoveries also represent species new to science. For example, in 1994 there were 1385 species of sponges described for the entire Australian fauna (including its external territories), with less than half of these known to live in tropical waters [6]. This knowledge took 200 years to acquire. By comparison, in 2001 the published taxonomic knowledge base of Australian sponge diversity had grown to over 1500 described species, but more importantly, our collections now contain more than 3000 species mainly from tropical waters, and most presumed to be new to science (i.e., they cannot currently be assigned to a published taxon). There has been a phenomenal advance in our marine biodiversity knowledge of this phylum, which would not have been possible without this program.

The formal processes of taxonomy (classifying and describing these species in the scientific literature), however, will take a lot longer than the discovery of these species. Nevertheless, our ability to discriminate these species as being new to science has already contributed significantly to marine biogeography, biodiversity, and conservation. Several of these findings have already been published (or are currently in press). Three significant discoveries on marine fauna have arisen to date.

Firstly, more than 600 species of sponges have been collected from the Great Barrier Reef (GBR) alone since 1993. Analysis of their taxonomic relationships [7], and ITS gene sequences of some key taxa [8], suggest that there are at least two distinct sponge faunas within the GBR (far south and central-north), and possibly a third distinct fauna (far north, although not yet substantially sampled), all of which appear to have minimal genetic contact. These new data have important implications to the development of management and conservation strategies of these unique marine resources, which have usually been assumed to represent a single GBR province. Similarly, from the same study, six distinct sponge faunas were discovered along the east coast, extending from Sydney to the Gulf of Carpentaria, 
each regional fauna containing many unique species (with a mean regional endemism of about $33 \%$ ) [7]. Previously, this entire east coast fauna was thought to consist only of two bioregions: a northern and a southern one.

Secondly, the AZGU program also provided an opportunity in 1997 to revisit the historic site of the first comprehensive study ever made of coral reefs anywhere in the world, the Low Isles, off Port Douglas in Far North Queensland. These isles were the site of the 1928-1929 Great Barrier Reef Expedition, led by Sir Maurice Yonge, which conducted extensive experiments and sampling of the coral reef biota. Much of our early knowledge of coral reef biodiversity, ecology, physiology, geology, etc., was derived from these early studies, and our own expedition was able to resurvey the sessile marine invertebrate fauna, to gauge potential changes to biodiversity over time (e.g., influence of human impacts on these popular reefs) [9].

Thirdly, these marine sponge collections have been instrumental in delineating major biodiversity "hotspots" throughout tropical Australia. This study was possible by analyzing the extensive marine collections using indicators of species richness, species uniqueness (endemism), and taxonomic affinities of these regional faunas in comparison with other regions in Southeast Asia and the western Pacific [10]. Based on a dataset of 2324 sponge species from 1343 investigated localities throughout tropical Australasia (which included datasets from studies pre-1993) 37 medium-scale (regional) faunas were recognized, each distinct in some way from adjacent regions (e.g., different species richness, different taxonomic composition, etc.). Of these regions five were "hotspots" of species richness (>250 spp/ region): two located on the northeast coast (southern and northern outer GBR), two in the Northern Territory (east and west sides of the 'Top End'), and one on the mid-northwest coast of Western Australia. Each of these regions contained about $27 \%$ of unique species, not found in other regions.

By combining some of the 37 medium-scale faunas according to their taxonomic affinities with each other, further analysis identified 13 larger-scale faunas (domains), of which three had >600 spp (outer GBR, Top End of the Northern Territory, mid-northwest coast of Western Australia), and were far richer than any other Australian marine province or domain, or the comparatively rich faunas of Vanuatu, Palau, and Thailand (which were used as outgroups for comparison). Each of these three Australian sponge "hotspots" contained distinctly different sponge faunas, with relatively few species common to all domains (a mean endemism of $47 \%$ ). These three domain-sized "hotspots" represent completely unique pools of genetic resources. Their existence also challenges our current simplistic marine biogeographic models of a widespread, northern "Tethyan fauna", with the erroneous presumption that these tropical genetic resources are homogeneous and widespread.

\section{New knowledge of the terrestrial flora}

Sixty plant species new to science have been discovered in the terrestrial flora collected since 1993. New populations of threatened species have been discovered in remote areas, providing the genetic material which can be used to propagate the species. Record of weed encroachment in native forests has been created for forest management use.

\section{Chemistry}

In an effort to find selective inhibitors of protein kinase enzymes, a marine sponge of the genus Ircinia yielded three new cheilanthane sesterterpenoids as well as lintenolide (2) previously reported from the sponge Cacospongia cf. linteiformis [11]. Cheilanthane sesterterpenenoids have not previously been reported from Ircinia species. The compounds were found to inhibit MSK1 (mitogen and stress-activated kinase) with an $\mathrm{IC}_{50}$ of $4 \mu \mathrm{M}$.

In continuing efforts to develop selective adenosine receptor agonists, a series of natural products have been discovered which display structure-activity within the various series. This information has been of value in docking studies to understand the binding domains.

(C) 2002 IUPAC, Pure and Applied Chemistry 74, 519-526 


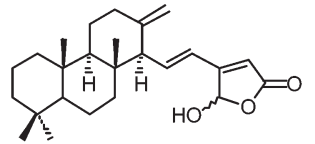

1

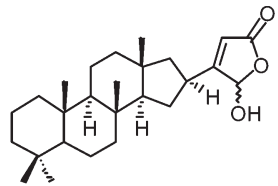

2

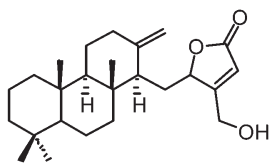

3

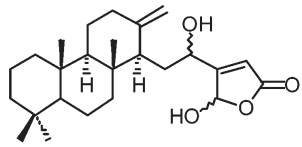

4

An extract of the Australian sea pen Anthoptilum cf. kukenthali, Hickson 1916, yielded five briarane diterpenes, anthoptilide A-E, the occurrence of a benzoate is unusual and the occurrence of a tiglate group in briaranes had not previously been reported [12]. The most potent compound was (7) with an $\mathrm{IC}_{50}$ of $3.1 \mu \mathrm{M},(\mathbf{6})$ was around ten-fold less potent at $45 \mu \mathrm{M}$ while $(\mathbf{5}, \mathbf{8})$ and (9) were an order of magnitude less potent at 420,500 , and $490 \mu \mathrm{M}$, respectively.

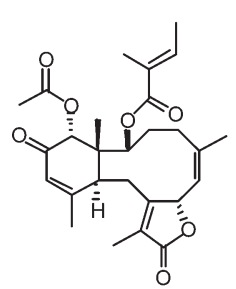

5

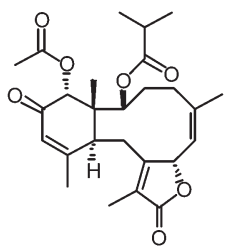

6

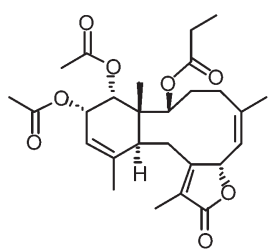

7

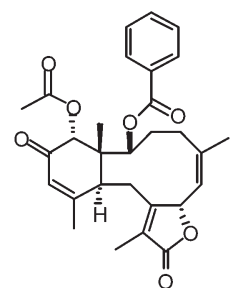

8

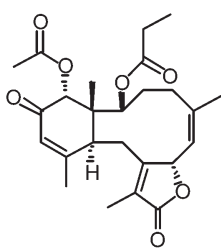

9

Psammaplin A (10) and psammaplin A 11'-sulfate (11) inhibited $\left[{ }^{3} \mathrm{H}\right] \mathrm{DPCPX}$ binding to rat brain adenosine $\mathrm{A}_{1}$ receptors with $\mathrm{IC}_{50}$ of 20 and $90 \mu \mathrm{M}$, respectively. In comparison, the dimer, bisaprasin 11 '-sulfate (12), did not show inhibition up to a concentration of $2.2 \mathrm{mM}$. The compounds were isolated from the marine sponge Aplysinella rhax [13]. Both of the sulfates, psammaplin A 11'-sulfate and bisaprasin 11'-sulfate, are previously unreported members of this class of bromotyrosines.<smiles>O=C(NO)NCCSCCNC(=O)C(Cc1ccc(O)c(Br)c1)NO</smiles>

$10 \quad \mathrm{R}=\mathrm{H}$

$11 \mathrm{R}=\mathrm{SO}_{3} \mathrm{Na}$

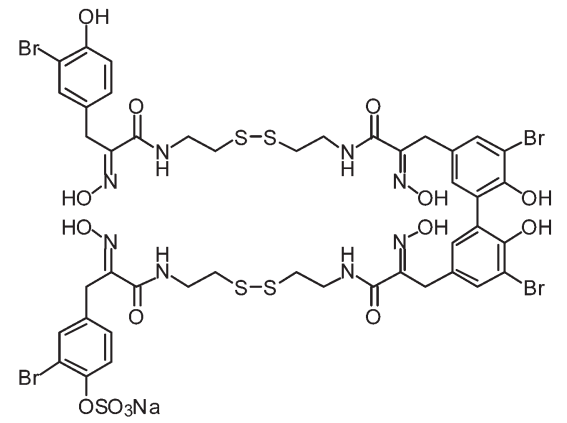

12

A soft coral of the genus Sarcophyton was found to contain two cembranes $(\mathbf{1 3}, \mathbf{1 4})$ that inhibited $\left[{ }^{3} \mathrm{H}\right] \mathrm{DPCPX}$ binding to rat brain adenosine at $300 \mu \mathrm{M}$, whereas the related cembranes $(\mathbf{1 5}, \mathbf{1 6})$ did not inhibit binding up to $300 \mathrm{mM}$. The conformational change may expose a different surface to the receptor.

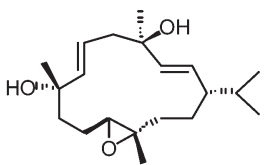

13

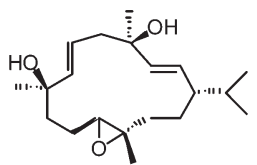

14

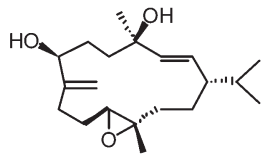

15

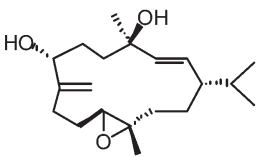

16 
We have isolated a series of polyoxygenated sterols that inhibit binding of $\left[\mathrm{I}^{125}\right]$ interleukin- 8 to the human CXCR1 (IL-8A) receptor [14]. The triol (17) had an $\mathrm{IC}_{50}$ of $20 \mu \mathrm{M}$, whereas the 3-acetate (18) had an $\mathrm{IC}_{50}$ of $5.5 \mu \mathrm{M}$ and the epoxide an $\mathrm{IC}_{50}$ of $4.5 \mu \mathrm{M}$.
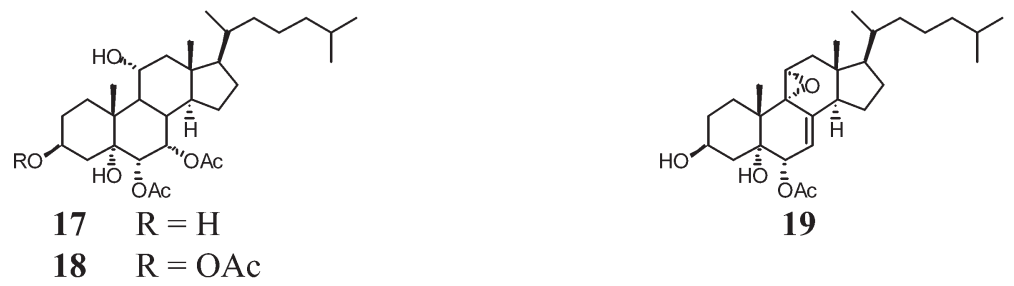

Chemokines play a central role in the chemoattraction of leukocytes during immune surveillance, inflammation, and the establishment of immunity. The chemokine receptors may play a role in chronic inflammation. In another study we identified embelin (19) that inhibited ( $\mathrm{IC}_{50} 2.4 \mu \mathrm{M}$ ) binding of MIP- $1 \alpha$ to HEK cell membranes expressing human CCR1 receptor. Methylation of embelin under basic conditions resulted in rearrangement to a series of cyclopentenones $(\mathbf{2 0 , 2 1 )}$ that also inhibited MIP- $1 \alpha$ binding ( $\mathrm{IC}_{50} 13$ and $11 \mu \mathrm{M}$, respectively).

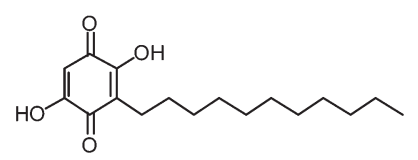

20

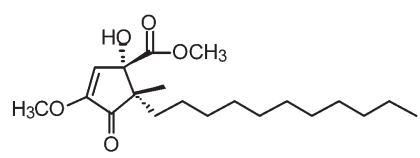

21

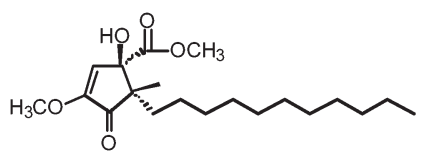

22

In concluding, this very successful AstraZeneca-Griffith University partnership is based on respect for relevant international laws and the Convention on Biological Diversity. Benefit sharing arrangements have been in place since 1993. As detailed in the manuscipt, significant benefits in conservation of biological diversity have been achieved.

\section{REFERENCES}

1. J. A. McNeely, K. R. Miller, W. V. Reid, R. A. Mittermeier, T. B. Werner. Conserving the World's Biological Diversity, p. 193, IUCN, Gland, Switzerland (1990).

2. G. M. Cragg, D. J. Newman, K. M. Snader. J. Nat. Prod. 60, 52 (1997).

3. T. Henkel, R. M. Brunne, H. Müller, F. Reichel. Angew. Chem., Int. Ed. Engl. 38, 643 (1999).

4. IMCRA. Interim Marine and Coastal Regionalisation for Australia, Version 3.3. Technical Committee of the Australian and New Zealand Environment and Conservation Council (1998) (http://www.ea.gov.au/coasts/mpa/nrsmpa/pubs/imcra.pdf).

5. M. H. G. Munro, J. W. Blunt, E. J. Dumdei, S. J. H. Hickford, R. E. Lill, S. Li, C. N. Battershill, A. R. Duchworth. J. Biotechnol. 70, 15 (1999).

6. J. N. A. Hooper and F. Wiedenmayer. In Zoological Catalogue of Australia, A. Wells (Ed.), Vol. 12, pp. 1-621, CSIRO, Melbourne (1994).

7. J. N. A. Hooper, J. A. Kennedy, S. E. List-Armitage, S. D. Cook, R. J. Quinn. Memoirs of the Queensland Museum 44, 263 (1999).

8. G. Woerheide, B. M. Degnan, J. N. A. Hooper. In Ninth International Coral Reef Symposium. World Coral Reefs in the New Millennium: Bridging Research and Management for Sustainable Development, D. Hopley, P. M. Hopley, J. Tamelander, T. Done (Eds.), p. 23, State Ministry for the Environment, Indonesia, Indonesian Institute of Sciences \& The International Society for Reef Studies, Bali, Indonesia (2000). 
9. J. N. A. Hooper, S. E. List-Armitage, J. A. Kennedy, S. D. Cook, C. A. Valentine. Memoirs of the Queensland Museum 44, 249 (1999).

10. J. N. A. Hooper, J. A. Kennedy, R. J. Quinn. Biodiversity and Conservation. In press.

11. M. S. Buchanan, A. Edser, G. King, J. Whitmore, R. J. Quinn. J. Nat. Prod. 64, 300 (2001).

12. N. B. Pham, M. S. Butler, P. C. Healy, R. J. Quinn. J. Nat. Prod. 63, 318 (2000).

13. N. B. Pham, M. S. Butler, R. J. Quinn. J. Nat. Prod. 63, 393 (2000).

14. P. d. A. Leone, J. Redburn, J. N. A. Hooper, R. J. Quinn. J. Nat. Prod. 63, 694 (2000). 